\title{
Skyrmion Control of Majorana States in Planar Josephson Junctions
}

\section{Narayan Mohanta ( $\nabla$ mohantan@ornl.gov )}

Oak Ridge National Laboratory https://orcid.org/0000-0003-3188-0445

\section{Satoshi Okamoto}

Oak Ridge National Laboratory https://orcid.org/0000-0002-0493-7568

\section{Elbio Dagotto}

University of Tennessee

\section{Article}

Keywords: MBS, SOC, Skyrmion, 2DEG

Posted Date: February 3rd, 2021

DOI: https://doi.org/10.21203/rs.3.rs-155447/v1

License: (c) (1) This work is licensed under a Creative Commons Attribution 4.0 International License. Read Full License

Version of Record: A version of this preprint was published at Communications Physics on July 15th, 2021. See the published version at https://doi.org/10.1038/s42005-021-00666-5. 


\title{
Skyrmion Control of Majorana States in Planar Josephson Junctions
}

\author{
Narayan Mohanta* ${ }^{1}$ Satoshi Okamoto, ${ }^{1}$ and Elbio Dagotto ${ }^{1,2}$ \\ ${ }^{1}$ Materials Science and Technology Division, Oak Ridge National Laboratory, Oak Ridge, TN 37831, USA \\ ${ }^{2}$ Department of Physics and Astronomy, The University of Tennessee, Knoxville, TN 37996, USA \\ *Email: mohantan@ornl.gov
}

\begin{abstract}
Planar Josephson junctions provide a versatile platform, alternative to the nanowire-based geometry, for the generation of the Majorana bound states, due to the additional phase tunability of the topological superconductivity. The proximity induction of chiral magnetism and superconductivity in a two-dimensional electron gas showed remarkable promises to manipulate topological superconductivity. Here, we consider a Josephson junction involving a skyrmion crystal and show that the chiral magnetism of the skyrmions can create and control the Majorana bound states without the requirement of an intrinsic Rashba spin-orbit coupling. Interestingly, the Majorana bound states in our geometry are realized robustly at zero phase difference at the junction. The skyrmion radius, being externally tunable by a magnetic field or a magnetic anisotropy, brings a unique control feature for the Majorana bound states.
\end{abstract}

\section{Introduction}

The unification of non-trivial spin texture and superconductivity via advanced interface engineering is a futuristic approach to create and manipulate non-Abelian Majorana bound states (MBS) for their controlled usage in fault-tolerant topological quantum computing ${ }^{1-5}$. The nanoscale control of magnetism not only relaxes the need for a specific form of Rashba spin-orbit coupling (SOC), but also motivates for a magnetic field-free platform for the braiding of the $\mathrm{MBS}^{6-12}$. Despite numerous successes in the search for the MBS in one-dimensional geometries, the associated limitations such as the intrinsic instabilities of one-dimensional systems, the need for fine tuning of parameters, and the technological obstacles in physical implementation, suggest to look for a two-dimensional platform ${ }^{13,14}$. The recent discovery of topological superconductivity in phase-controlled planar Josephson junctions (JJs) is, therefore, a major step towards the realization of a two-dimensional array of MBS for designing scalable braiding protocols ${ }^{15-18}$. The JJ geometry provides additional control to tune the MBS by changing the shape of the junction, strain and unconventional SOC ${ }^{19-22}$. Previous works on the JJ-based platforms, however, revealed the requirements of a strong intrinsic Rashba SOC and $\pi$-phase biasing of the JJ. These constraints pose serious challenges in the detection and manipulation of the MBS under realistic conditions. Chiral magnetism in proximity to an $s$-wave superconductor generates exotic effects including the appearance of the Majorana modes ${ }^{23-28}$; however, the location and stability of the Majorana states in these platforms are difficult to anticipate due to their non-localized nature.

In our considered geometry, the planar JJ, composed of a two-dimensional electron gas (2DEG) and an $s$-wave superconductor, is placed on top of a Néel-type skyrmion crystal (SkX) in such a way that the 2DEG experiences the spatially-varying magnetic field from the bottom SkX and it is also proximitized to the electron pairing from the top superconductors, as described in Fig. 1a. The interplay between the SkX spin texture and the proximityinduced superconductivity leads to topological supercon- ductivity near the middle quasi-one-dimensional channel of the JJ with localized MBS at its two ends. The advantages of using the SkX are: (i) the chiral magnetism generates a robust SOC and a local Zeeman field which remove the stringent criteria of a strong Rashba-type SOC and, therefore, essentially expands the region of parameter space to realize the MBS, (ii) the existence of the MBS can be further controlled externally by tuning the skyrmion radius, and (iii) usual planar JJs are required to be phase biased with a phase difference $\varphi=\pi$, between the two superconducting regions, to minimize the critical magnetic field for the topological transition and to maximize the chemical-potential range within which the MBS appear ${ }^{15}$; the current SkX-based JJ is not required to be phase biased and the MBS can be found robustly at $\varphi=0$. Using the zero-energy feature of the quasiparticle states with a topological energy gap, sharp localization of these states, charge-neutrality condition, two order parameters, viz. Majorana polarization and curvature of the density of states, we confirm the existence of the MBS in our set up. The tunable phase difference and the skyrmion radius together provide broad, flexible control of the MBS which is indispensable to achieve the long-sought-after goal of the braiding.

\section{Results}

Theoretical set up. For the generation of the SkX, we consider a heterointerface of a thin-layer ferromagnet $(\mathrm{FM})$ and a heavy metal or a heavy insulator $(\mathrm{HM} / \mathrm{HI})$. The strong SOC in the $\mathrm{HM} / \mathrm{HI}$ gives rise to a large Dzyaloshinskii-Moriya interaction (DMI) at the interface between the FM and the HM/HI. The cooperation between the DMI and the ferromagnetic exchange interaction of the FM produces a triangular SkX, in the presence of a magnetic field or an anisotropy. Our Monte Carlo simulations reveal that columns of skyrmions, arranged in a triangular array, appear spontaneously within a sixlayer FM, although the DMI exists predominantly at the interface between the FM and the HM/HI, as shown in Fig. 1b. We use Metropolis energy minimization tech- 


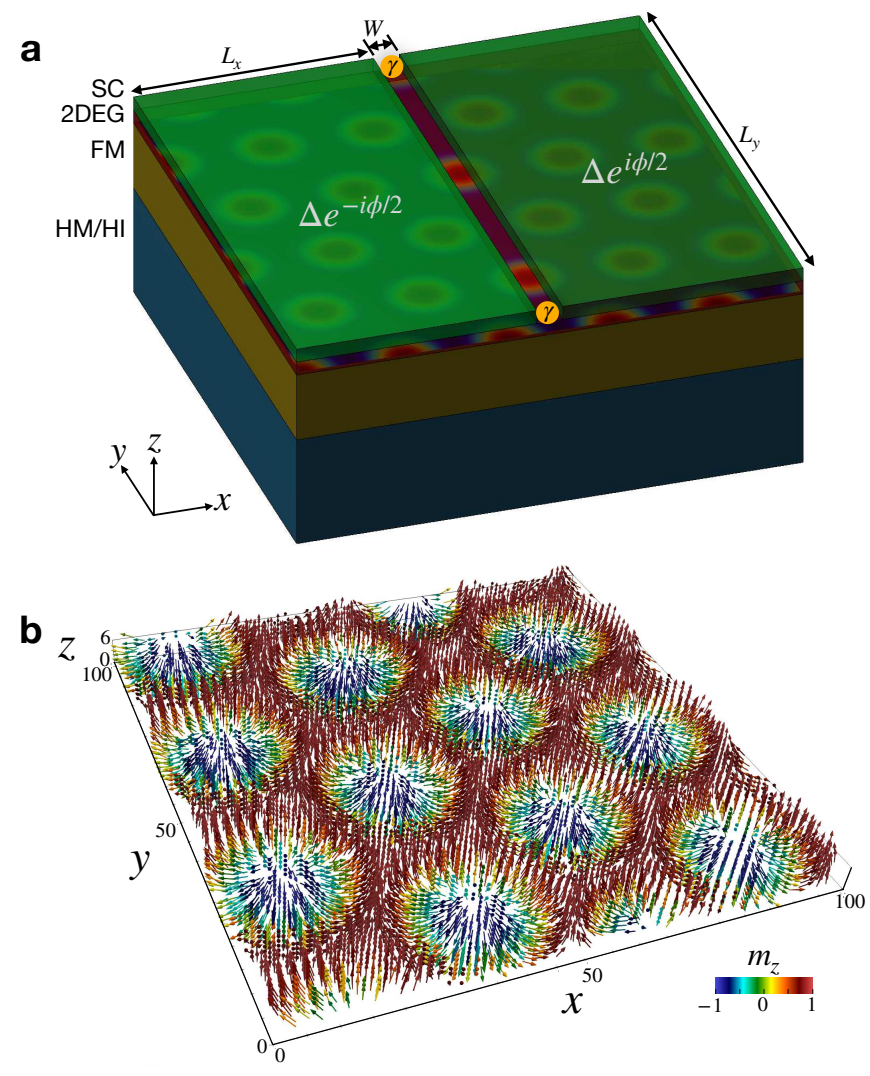

FIG. 1. Device geometry and a skyrmion crystal. a. Planar Josephson junction on top of a skyrmion crystal (SkX). The two-dimensional electron gas (2DEG) exhibits both proximity-induced superconductivity from the top superconductor (SC) layers and spatially-varying magnetism from the bottom SkX. The SkX is spontaneously created in the ferromagnet (FM) due to the competition between exchange interactions in the FM and the heavy metal or heavy insulator $(\mathrm{HM} / \mathrm{HI})$, with a field or anisotropy. The zeroenergy Majorana bound states (yellow bubbles) are localized at the two ends of the quasi-one-dimensional metallic channel. b. The SkX spin texture, spontaneously generated in a Monte Carlo simulation using a $100 \times 100 \times 6$ lattice with ferromagnetic exchange interaction strength $J=1$, DMI strength $D=0.3 J$, magnetic field $H_{z}=0.1 J$, spin amplitude $S=1$, and easy-plane anisotropy $A=0.01 J$.

nique formulated with the following Hamiltonian

$$
\begin{aligned}
\mathcal{H}= & -J \sum_{\langle i j\rangle} \mathbf{S}_{i} \cdot \mathbf{S}_{j}-D \sum_{\langle a b\rangle}\left(\hat{z} \times \hat{r}_{a b}\right) \cdot\left(\mathbf{S}_{a} \times \mathbf{S}_{b}\right) \\
& -H_{z} \sum_{i} S_{z i}-A \sum_{a}\left|S_{z a}\right|^{2}
\end{aligned}
$$

where $J$ is the nearest-neighbor ferromagnetic exchange interaction strength in the FM, $D$ is the DMI strength at the bottom FM layer that interfaces with the HM/HI, $H_{z}$ is the perpendicular magnetic field, $A$ is the easyplane magnetic anisotropy at the bottom FM layer, $i, j$ are the site indices in the entire $\mathrm{FM}$, and $a, b$ are the twodimensional site indices at the bottom FM layer. The DMI, present dominantly at the interface between the FM and the HM/HI, generates a Néel-type $\mathrm{SkX}^{29-31}$.
Besides the engineered interfaces, the SkX naturally appears in a wide variety of materials ${ }^{32-35}$ that can also be utilized in the proposed device geometry, instead of the combination of the FM and the HM/HI. Also, the SkX can be artificially-created without the need for any external magnetic field by nanopatternization ${ }^{36}$.

The spin texture $\mathbf{B}_{i}$ on the top layer of the FM, obtained from the Monte Carlo simulations, is used to obtain the low-energy spectrum of the planar JJ by solving self-consistently the Bogoliubov-de Gennes equations. The proximity-induced superconductivity in the 2DEG, which is subject to the $\mathrm{SkX}$ spin texture $\mathbf{B}_{i}$, is described by the Hamiltonian

$$
\begin{aligned}
& \mathcal{H}_{\mathrm{BdG}}=-t \sum_{\langle i j\rangle, \sigma}\left(c_{i \sigma}^{\dagger} c_{j \sigma}+H . c .\right)+\sum_{i, \sigma}(4 t-\mu) c_{i \sigma}^{\dagger} c_{i \sigma} \\
& -\frac{1}{2} g \mu_{B} \sum_{i, \sigma}\left(\mathbf{B}_{i} \cdot \boldsymbol{\sigma}\right)_{\sigma \sigma^{\prime}} c_{i \sigma}^{\dagger} c_{i \sigma^{\prime}}+\sum_{i}\left(\Delta_{i} c_{i \uparrow}^{\dagger} c_{i \downarrow}^{\dagger}+H . c .\right),
\end{aligned}
$$

where $t=\hbar^{2} /\left(2 m^{*} a^{2}\right)$ is the hopping energy, $m^{*}$ is the effective mass of electrons, $a$ is the unit spacing of the lattice grid, $\mu$ is the chemical potential, and $\Delta_{i}$ is the induced local $s$-wave pairing amplitude on the two sides of the JJ that are attached to the top $\mathrm{Al}$ layer. The pairing amplitude $\Delta_{i}=-U_{i}\left\langle c_{i \uparrow} c_{i \downarrow}\right\rangle$ is calculated self-consistently using the onsite attractive interaction strength $U_{i}$ of the induced superconducting states in the 2DEG. $U_{i}=U$ in the 2DEG below the Al superconductors and zero in the middle metallic channel. The value $U=2 \mathrm{meV}$ is determined by setting $\Delta_{i}=0.2 \mathrm{meV}$, the estimated proximity-induced gap magnitude for a $2 \mathrm{DEG}$ with an SC interface ${ }^{37,38}$, without any spin texture. The $g$ factor and the effective mass are set to $g=50$ and $m^{*}=0.017 m_{0}$ for $\mathrm{InSb}^{39,40}$. The lattice grid spacing used is $a=10 \mathrm{~nm}^{41}$ with which the hopping energy becomes $t=22.44 \mathrm{meV}$. The amplitude of the spin texture $\mathbf{B}_{i}$ is set to $B_{0}=0.3 \mathrm{~T}$, compatible with the saturation magnetization $M_{s}=1.7 \times 10^{6} \mathrm{~A} / \mathrm{m}$ for $\mathrm{CoFe}^{10,11}$. We present results for a planar JJ with length $L_{y}=2 \mu \mathrm{m}$, transverse length of the SC leads $L_{x}=200 \mathrm{~nm}$, and width of the quasi-one-dimensional metallic channel $W=50 \mathrm{~nm}$.

Emergence of the MBS. The low-energy spectrum, shown in Fig. 2a, reveals that there exist multiple ranges of the chemical potential within which the zero-energy MBS appear. To determine the Majorana character of the quasiparticle states, we compute the Majorana polarization, defined as $^{42}$

$$
\mathcal{P}_{\mathcal{M}, n}=2 \sum_{i}\left|u_{i \downarrow}^{n} v_{i \downarrow}^{n *}-u_{i \uparrow}^{n} v_{i \uparrow}^{n *}\right|
$$

where $u_{i \uparrow}^{n}$ and $v_{i \uparrow}^{n}$ are the Bogoliubov-de Gennes quasiparticle and quasihole amplitudes, respectively, corresponding to the $n^{\text {th }}$ eigenstate, spin $\uparrow$, and site $i$. As evident from Fig. $2 \mathbf{a}, \mathcal{P}_{\mathcal{M}, n} \gtrsim 1$ indicates the occurrence of robust MBS with a finite topological energy gap. The Majorana polarization $\mathcal{P}_{\mathcal{M}}$, plotted with $\mu$ in Fig. $2 \mathbf{b}$, acquires finite values within the range of $\mu$, in which the MBS emerge. The delta function-like peaks in $\mathcal{P}_{\mathcal{M}}$ are 


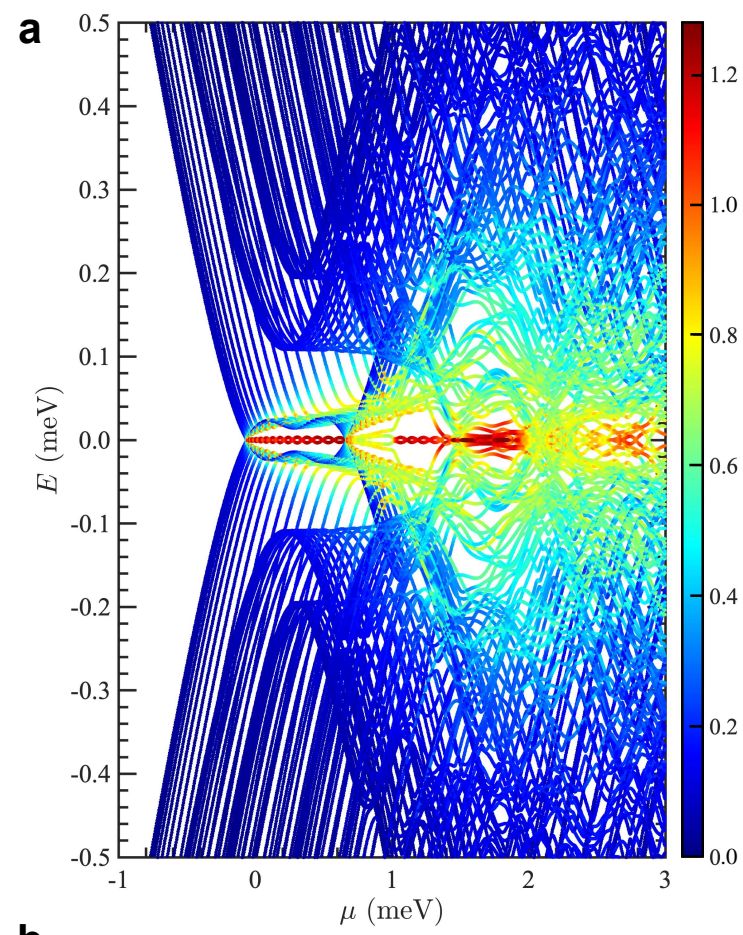

b
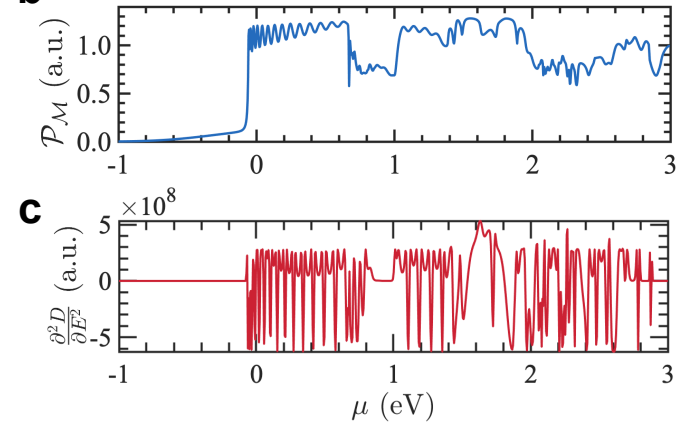
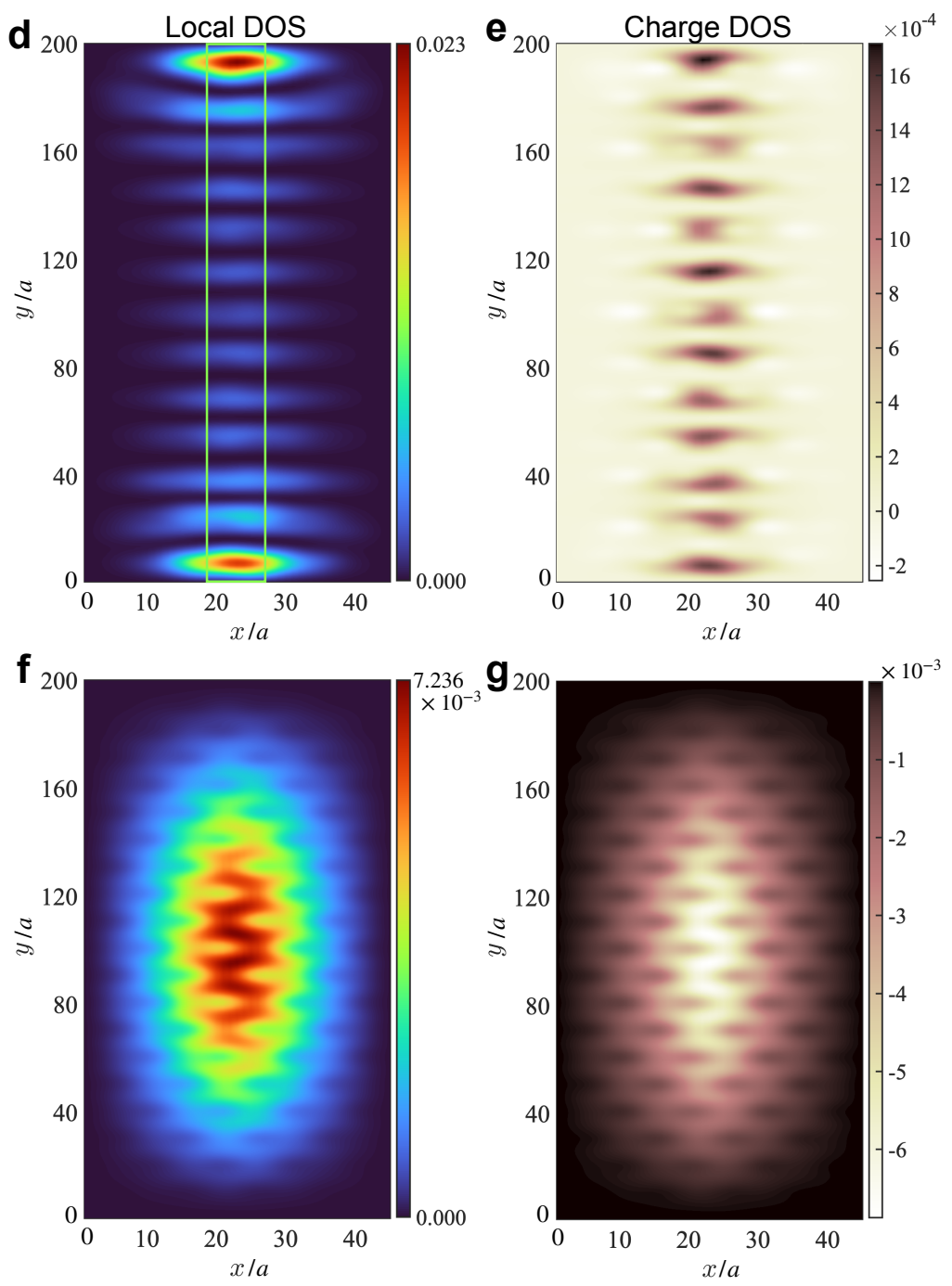

FIG. 2. Emergence of the Majorana bound states with changing chemical potential. a. The quasiparticle spectrum of the planar Josephson junction at phase difference $\varphi=0$ with varying chemical potential $(\mu)$, showing the emergence of the zero-energy MBS. The colorbar represents the Majorana polarization $\mathcal{P}_{\mathcal{M}}$ that displays the Majorana character of the quasiparticle states. The SkX with a skyrmion diameter $D_{s k}=10 a$ was obtained using a magnetic field $H_{z}=0.95 J$ and a DMI strength $D=1.6 J$ in the Monte Carlo calculations. b., c. The Majorana polarization $\mathcal{P}_{\mathcal{M}}$ and the curvature of the density of states at zero energy $\frac{\partial^{2} D}{\partial E^{2}}$, with varying $\mu$, showing the $\mu$ range within which the MBS appear. The delta-function-like peaks are associated with the oscillations of the MBS with changing $\mu$. d. e. The profiles of the local density of states and the charge density of states at $\mu=0.5 \mathrm{meV}$. The green rectangle in $\mathbf{d}$ indicates the quasi-one-dimensional metallic channel of the planar Josephson junction at the ends of which the MBS appear. f., g. The profiles of the local density of states and the charge density of states at $\mu=-0.5 \mathrm{meV}$, in the non-topological regime.

the signatures of the Majorana oscillations, which is also clearly seen in the low-energy spectrum in Fig. 2a, originating due to the overlap of the MBS wave functions at the two ends of the finite-length quasi-one-dimensional channel. The Majorana oscillations in $\mathcal{P}_{\mathcal{M}}$ have also been confirmed from the calculations of a one-dimensional wire (see Supplementary Information). The Majorana polarization in this planar JJ geometry can be probed using spin-selective Andreev reflection technique ${ }^{43}$. To further characterize the evolution of the topological superconductivity with changing a parameter, such as $\mu$, we look at the curvature of the density of states at zero energy $\frac{\partial^{2} D}{\partial E^{2}}$, where $D(E)$ is defined as $^{44}$

$$
D(E)=\sum_{i, n, \sigma}\left(\left|u_{i \sigma}^{n}\right|^{2}+\left|v_{i \sigma}^{n}\right|^{2}\right) \delta\left(E-E_{n}\right),
$$

and $\delta\left(E-E_{n}\right)$ is modeled using a Gaussian with broadening $0.001 \mathrm{meV}(\ll t)$. The second derivative is computed using the second-order finite-difference method. These two quantities, $\mathcal{P}_{\mathcal{M}}$ and $\frac{\partial^{2} D}{\partial E^{2}}$, may provide additional insight in the experimental detection of the MBS, besides the conventional zero-bias conductance peak ${ }^{45}$ which often leads to ambiguity due to other possible zero-bias states in a superconductor ${ }^{46}$. 

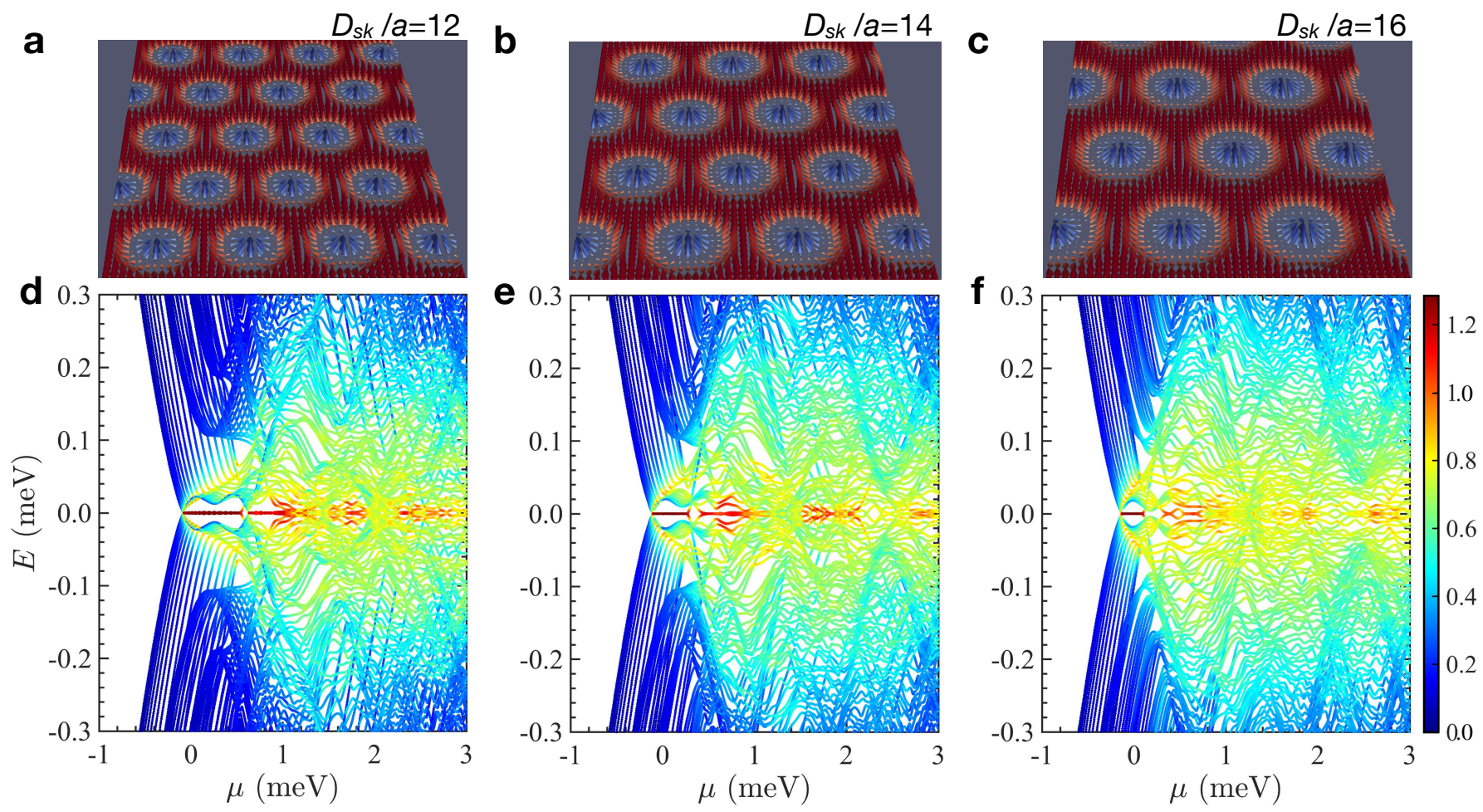

FIG. 3. Skyrmion tuning of the Majorana bound states. a-c The skyrmion crystals of different skyrmion diameters a. $D_{s k}=12 a$, b. $D_{s k}=14 a$, c. $D_{s k}=16 a$, obtained in the Monte Carlo calculations using a. a magnetic field $H_{z}=0.8 J$, a DMI strength $D=1.4 J$, b. $H_{z}=0.45 J, D=J$, and c. $H_{z}=0.23 J, D=0.6 J$. d-f The corresponding quasiparticle spectra of the planar Josephson junction at the phase difference $\varphi=0$ with varying chemical potential, obtained by solving the Bogoliubov-de Gennes equations with the above skyrmion crystal spin configurations. The colorbar in d-f represents the Majorana polarization $\mathcal{P}_{\mathcal{M}}$ of the quasiparticle states.

As shown in Fig. 2c, $\frac{\partial^{2} D}{\partial E^{2}}$ takes finite values in the same ranges of $\mu$ as that of the Majorana polarization $\mathcal{P}_{\mathcal{M}}$. The Majorana oscillations, in the form of deltafunction-like peaks, is also noticeable in $\frac{\partial^{2} D}{\partial E^{2}}$, albeit with changes in the sign. To visualize the location of the zero-energy MBS, we show, in Fig. 2d, the profile of the local density of states $\rho_{\mathrm{LDOS}}^{i}=\sum_{\sigma}\left(\left|u_{i \sigma}\right|^{2}+\left|v_{i \sigma}\right|^{2}\right)$, corresponding to the lowest positive-energy eigenstate at $\mu=0.5 \mathrm{meV}$ where the JJ is in the topological superconducting regime. The sharp peaks in $\rho_{\mathrm{LDOS}}^{i}$ indicate that the MBS are localized predominantly near the two ends of the quasi-one-dimensional channel. Figure 2e shows the profile of the charge density of states $\rho_{\mathrm{CDOS}}^{i}=\sum_{\sigma}\left(\left|u_{i \sigma}\right|^{2}-\left|v_{i \sigma}\right|^{2}\right)$ corresponding to the lowest positive-energy eigenstate at $\mu=0.5 \mathrm{meV}$. The profiles of $\rho_{\mathrm{LDOS}}^{i}$ and $\rho_{\mathrm{CDOS}}^{i}$, at $\mu=-0.5 \mathrm{meV}$ where the JJ is in the topologically-trivial superconducting regime, are shown in Fig. $2 \mathbf{f}$ and Fig. 2g, respectively. In this case, both the quasiparticle state and the charge density are distributed near the middle of the quasi-one-dimensional channel. Interestingly, a comparison of Fig. 2e and Fig. 2g, implies an order-of-magnitude suppression in $\rho_{\mathrm{CDOS}}^{i}$ which is reminiscent of the local charge-neutrality signature of the MBS and is another confirmation of the Majorana character of this state. The above results establish that the SOC, generated by the SkX, alone can lead to the emergence of the MBS in the planar JJ devices.

Skyrmion tuning of the MBS. The skyrmion size in a $\mathrm{SkX}$ is tunable, with remarkable precession, by an external magnetic field, magnetic anisotropy and advanced symmetry protocol at heterointerfaces ${ }^{47-49}$. In our Monte Carlo simulations, the skyrmion size was varied by tuning the magnetic field and the DMI, as shown in Figs. 3a-c. The Bogoliubov-de Gennes quasiparticle spectra at different skyrmion sizes, shown in Figs. 3df, imply that the presence of the zero energy MBS at a given chemical potential can be turned ON or OFF by only changing the skyrmion properties. The minimum diameter of the skyrmions, realized in our Monte Carlo simulations, is 10 lattice spacings for which the MBS appear in the discussed planar JJ setting. With increasing the skyrmion size, we find that the range of the chemical potential within which the MBS appear decreases effectively, however, the oscillation amplitude of the MBS is suppressed gradually. Therefore, the skyrmions offer a unique ability to manipulate the localization length of the MBS in the planar JJs. The strongly-localized MBS in the skyrmion-tuned planar JJs can, therefore, have advantageous over other platforms for MBS realization in fault-tolerant topological quantum computing.

The broken inversion symmetry at the interface between the 2DEG and the superconductor, often leads 
to a sizable intrinsic Rashba SOC which is usually considered as the primary mechanism for modifying the pairing symmetry of the induced superconductivity ${ }^{50,51}$, leading to the desired topological superconductivity. We find that the MBS remain robust in the presence of the intrinsic Rashba SOC (see Supplementary Information).

Phase tuning of the MBS. Another important control parameter, that sets the planar JJs apart from other platforms hosting the MBS, is the phase difference $\varphi$ between the two superconducting regions of a JJ. The theoretical prediction $^{15}$ and the subsequent experimental discoveries $^{16,17}$ suggest that the JJ needs to be biased by a phase difference $\varphi=\pi$ to minimize the critical Zeeman field, required for inducing the topological superconductivity. Remarkably, in the current JJ set up with the SkX, the topological superconductivity is induced at $\varphi=0$, as depicted by the quasiparticle spectrum with varying $\varphi$ in Fig. 4a. With increasing $\varphi$, the MBS move gradually from zero to higher energies, indicating an enhancement in the localization length of the MBS. The MBS appear again at zero energy above $\varphi \approx 3 \pi / 2$.

This dephasing effect of the MBS can be understood from the Majorana oscillations - as we find that the oscillation increases with increasing $\varphi$ in the range $0<\varphi \leq \pi$ (see Supplementary Information). The finite length of the quasi-one-dimensional metallic channel gives rise to the oscillations of the zero-energy MBS with varying chemical potential $\mu$. Furthermore, the finite width of the metallic channel provides extra room for delocalization of the MBS at the two ends, contributing additively to the Majorana oscillation. The middle metallic region of the JJ can be perceived as a quasi-one-dimensional void region surrounded by the superconducting 2DEG. Additional phase difference between the two superconducting sides, therefore, only causes disruption to the induced topological superconductivity. This phenomenon generically takes place at several values of the chemical potential, as shown in Fig. 4b, where we plot the Majorana polarization $\mathcal{P}_{\mathcal{M}}$ of the lowest positive energy eigenstate in the parameter space spanned by the phase difference $\varphi$ and the chemical potential $\mu$. For the chosen range of $\mu$ values, the Majorana polarization decreases substantially within the range $\pi / 2 \lesssim \varphi \lesssim 3 \pi / 2$. The Majorana oscillation in $\mathcal{P}_{\mathcal{M}}$, however, survives up to $\varphi \approx \pi$. At $\varphi=\pi$, $\mathcal{P}_{\mathcal{M}}$ vanishes completely, indicating the disappearance of the MBS. Therefore, $\varphi=0$ is the most favorable condition to realize the MBS in our JJ set up and the phase difference can be further tuned to control the presence of the MBS.

\section{Discussion}

The skyrmions bring outstanding control functionalities to the planar JJs for the creation and manipulation of the zero-energy MBS and their localization properties. The SkXs, being realized in an abundance of magnetic materials and also artificially created in patterned magnetic materials, offer a novel, feasible approach for
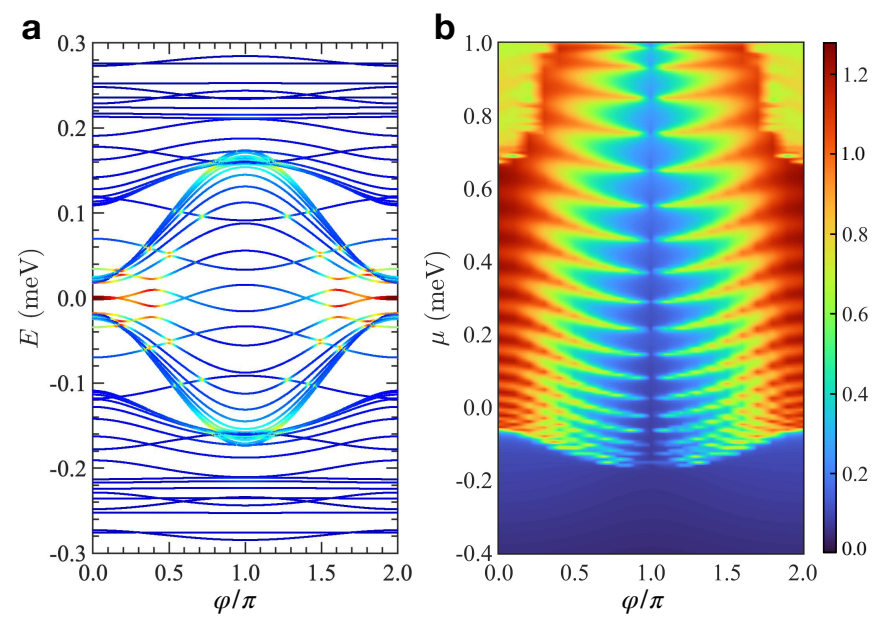

FIG. 4. Phase tuning of the Majorana bound states. a. Quasiparticle spectrum of the planar Josephson junction at a chemical potential $\mu=0.5 \mathrm{meV}$ with changing phase difference $\varphi$ between the two superconducting regions. The skyrmion diameter of the skyrmion crystal was $D_{s k}=10 a$. b. Colormap of the Majorana polarization of the lowest positive energy state in the plane spanned by the phase difference $\varphi$ and the chemical potential $\mu$. The Majorana bound states appear in the parameter regimes with $\mathcal{P}_{\mathcal{M}} \geq 1, \varphi=0$ being the most favorable scenario in this set up.

advanced manipulation of the zero-energy MBS. The proposed planar JJ, combined with a SkX, has the major advantages that there is no need for a strong intrinsic Rashba-type SOC and phase-biasing constraint for the realization of the zero-energy MBS. The enhanced tunability of the MBS in the proposed two-dimensional platform opens up opportunities for designing new MBS braiding protocols for the fault-tolerant topological quantum computing, and investigating Majorana spectroscopy using the multi-terminal superconducting quantum interference devices.

\section{Methods}

Monte Carlo simulations. The SkX spin configurations were obtained using a $L_{x} \times L_{y} \times L_{z}$ lattice with periodic boundary conditions along the $x$ and $y$ directions and open boundary conditions along the $z$ direction. A bias-free sampling method, that provides a full and uniform coverage of the phase space spanned by the spin angles, was used for generating the completely random spin configurations. The calculation was started at a high temperature $T=10 \mathrm{~J}$ with a random spin configuration and the temperature was lowered slowly down to a low value $T=0.001 J$ in 2000 steps. At each temperature step, $10^{10}$ Monte Carlo spin updates were performed. In each spin update step, a new spin direction was chosen randomly within a small cone spanned around the initial spin direction. The new spin configuration was accepted or rejected according to the Metropolis energy-minimization algorithm by comparing the total energies of the previous and the new trial spin configurations, calculated using the Hamiltonian (1).

Self-consistent Bogoliubov-de Gennes formalism. The Hamiltonian (2), which is quadratic in the fermionic opera- 
tors $\hat{c}_{i \sigma}$, can be solved by exact diagonalization via a unitary transformation $\hat{c}_{i \sigma}=\sum_{n} u_{i \sigma}^{n} \hat{\gamma}_{n}+v_{i \sigma}^{n *} \hat{\gamma}_{n}^{\dagger}$, where $\hat{\gamma}_{n}^{\dagger}\left(\hat{\gamma}_{n}\right)$ is a fermionic creation (annihilation) operator of the quasiparticle/quasiphole state in the $n^{\text {th }}$ energy eigenstate. The quasiparticle amplitudes $u_{i \sigma}^{n}$ and the quasihole amplitudes $u_{i \sigma}^{n}$ are determined by solving the Bogoliubov-de Gennes equations: $\sum_{j} \mathcal{H}_{i j} \psi_{j}^{n}=\epsilon_{n} \psi_{n}^{i}$, where $\psi_{i}^{n}=\left[u_{i \uparrow}^{n}, u_{i \downarrow}^{n}, v_{i \uparrow}^{n}, v_{i \downarrow}^{n}\right]^{T}$ is the basis wave function and $\epsilon_{n}$ is the $n^{\text {th }}$ energy eigenvalue. The Hamiltonian $\mathcal{H}_{i j}$ is expressed in the following matrix form

$$
\mathcal{H}_{i j}=\left(\begin{array}{cccc}
\mathcal{H}_{\uparrow \uparrow} & \mathcal{H}_{\uparrow \downarrow} & 0 & \Delta_{i} \\
\mathcal{H}_{\downarrow \uparrow} & \mathcal{H}_{\downarrow \downarrow} & -\Delta_{i} & 0 \\
0 & -\Delta_{i}^{*} & -\mathcal{H}_{\uparrow \uparrow}^{*} & -\mathcal{H}_{\uparrow \downarrow}^{*} \\
\Delta_{i}^{*} & 0 & -\mathcal{H}_{\downarrow \uparrow}^{*} & -\mathcal{H}_{\downarrow \downarrow}^{*}
\end{array}\right),
$$

where $\mathcal{H}_{\uparrow \uparrow, \downarrow \downarrow}=-t\left(1-\delta_{i j}\right)+(4 t-\mu) \delta_{i j}-1 / 2 \sigma g \mu_{B} B_{z}$, where $\sigma= \pm$ for $\uparrow \uparrow, ~ \downarrow \downarrow$, and $\mathcal{H}_{\uparrow \downarrow}=-1 / 2 g \mu_{B}\left(B_{x}+i B_{y}\right)$. The $s$-wave pairing amplitude $\Delta_{i}=-U_{i}\left\langle c_{i \uparrow} c_{i \downarrow}\right\rangle$ is computed using $\Delta_{i}=-U_{i} \sum_{n}\left[u_{i \uparrow}^{n} v_{i \downarrow}^{n *}\left(1-f\left(\epsilon_{n}\right)\right)+u_{i \downarrow}^{n} v_{i \uparrow}^{n *} f\left(\epsilon_{n}\right)\right]$, where $U_{i}=U$ inside the two superconducting regions and zero in the middle quasi-one-dimensional metallic channel, $f\left(\epsilon_{n}\right)=1 /\left(1+e^{\epsilon_{n} / k_{B} T}\right)$ is the Fermi-Dirac distribution function at temperature $T$. The self-consistency iterations were performed until the pairing amplitudes $\Delta_{i}$ were converged at every lattice sites.

\section{Data availability}

The data presented in this paper are available from the corresponding author upon reasonable request.

\section{Code availability}

Codes used in this paper are available from the corresponding author upon reasonable request.

\section{REFERENCES}

1 A.Yu. Kitaev, "Fault-tolerant quantum computation by anyons," Ann. Phys. 303, 2 (2003).

2 M. M. Desjardins, L. C. Contamin, M. R. Delbecq, M. C. Dartiailh, L. E. Bruhat, T. Cubaynes, J. J. Viennot, F. Mallet, S. Rohart, A. Thiaville, A. Cottet, and T. Kontos, "Synthetic spin-orbit interaction for Majorana devices," Nat. Mater. 18, 1060-1064 (2019).

3 A. Yazdani, "Conjuring Majorana with synthetic magnetism," Nat. Mater. 18, 1036 (2019).

${ }^{4}$ C. Nayak, S. H. Simon, A. Stern, M. Freedman, and S. Das Sarma, "Non-abelian anyons and topological quantum computation," Rev. Mod. Phys. 80, 1083-1159 (2008).

5 J. Alicea, Y. Oreg, G. Refael, F. von Oppen, and M. P. A. Fisher, "Non-Abelian statistics and topological quantum information processing in 1D wire networks," Nat. Phys. 7, 412 (2011).

${ }^{6}$ M. Kjaergaard, K. Wölms, and K. Flensberg, "Majorana fermions in superconducting nanowires without spin-orbit coupling," Phys. Rev. B 85, 020503 (2012).

7 J. Klinovaja, P. Stano, A. Yazdani, and D. Loss, "Topological superconductivity and Majorana fermions in RKKY systems," Phys. Rev. Lett. 111, 186805 (2013).

8 J. Klinovaja and D. Loss, "Giant spin-orbit interaction due to rotating magnetic fields in Graphene nanoribbons," Phys. Rev. X 3, 011008 (2013).
9 G. L. Fatin, A. Matos-Abiague, B. Scharf, and I. Žutić, "Wireless Majorana bound states: From magnetic tunability to braiding," Phys. Rev. Lett. 117, 077002 (2016).

10 N. Mohanta, T. Zhou, J.-W. Xu, J. E. Han, A. D. Kent, J. Shabani, I. Žutić, and Alex Matos-Abiague, "Electrical Control of Majorana Bound States Using Magnetic Stripes," Phys. Rev. Applied 12, 034048 (2019).

11 T. Zhou, N. Mohanta, J. E. Han, A. Matos-Abiague, and I. Žutić, "Tunable magnetic textures in spin valves: From spintronics to Majorana bound states," Phys. Rev. B 99, 134505 (2019).

12 J. Herbrych, M. Środa, G. Alvarez, M. Mierzejewski, and E. Dagotto, "Interaction-induced topological phase transition and Majorana edge states in low-dimensional orbitalselective Mott insulators," arXiv:2011.05646 (2020).

13 J. Shabani, M. Kjaergaard, H. J. Suominen, Younghyun Kim, F. Nichele, K. Pakrouski, T. Stankevic, R. M. Lutchyn, P. Krogstrup, R. Feidenhans'l, S. Kraemer, C. Nayak, M. Troyer, C. M. Marcus, and C. J. Palmstrøm, "Two-dimensional epitaxial superconductor-semiconductor heterostructures: A platform for topological superconducting networks," Phys. Rev. B 93, 155402 (2016).

14 T. Karzig, C. Knapp, R. M. Lutchyn, P. Bonderson, M. B. Hastings, C. Nayak, J. Alicea, K. Flensberg, S. Plugge, Y. Oreg, C. M. Marcus, and M. H. Freedman, "Scalable designs for quasiparticle-poisoning-protected topological quantum computation with Majorana zero modes," Phys. Rev. B 95, 235305 (2017).

15 F. Pientka, A. Keselman, E. Berg, A. Yacoby, A. Stern, and B. I. Halperin, "Topological Superconductivity in a Planar Josephson Junction," Phys. Rev. X 7, 021032 (2017).

16 H. Ren, F. Pientka, S. Hart, A. T. Pierce, M. Kosowsky, L. Lunczer, R. Schlereth, B. Scharf, E. M. Hankiewicz, L. W. Molenkamp, B. I. Halperin, and A. Yacoby, "Topological superconductivity in a phase-controlled Josephson junction," Nature 569, 93 (2019).

17 A. Fornieri, A. M. Whiticar, F. Setiawan, E. Portolés, A. C. C. Drachmann, A. Keselman, S. Gronin, C. Thomas, T. Wang, R. Kallaher, G. C. Gardner, E. Berg, M. J. Manfra, A. Stern, C. M. Marcus, and F. Nichele, "Evidence of topological superconductivity in planar Josephson junctions," Nature 569, 89 (2019).

18 W. Mayer, M. C. Dartiailh, J. Yuan, K. S. Wickramasinghe, A. Matos-Abiague, I. Žutić, and J. Shabani, "Phase signature of topological transition in Josephson Junctions," arXiv:1906.01179 (2019).

19 T. Zhou, M. C. Dartiailh, W. Mayer, J. E. Han, A. MatosAbiague, J. Shabani, and I. Žutić, "Phase control of Majorana bound states in a topological X junction," Phys. Rev. Lett. 124, 137001 (2020).

20 M. Alidoust, M. Willatzen, and A.-P. Jauho, "Strainengineered Majorana zero energy modes and $\varphi_{0}$ Josephson state in black phosphorus," Phys. Rev. B 98, 085414 (2018).

21 M. Alidoust, C. Shen, and I. Zutic, "Cubic spin-orbit coupling and anomalous Josephson effect in planar junctions," arXiv:2101.08272 (2021).

22 F. Setiawan, A. Stern, and E. Berg, "Topological superconductivity in planar Josephson junctions: Narrowing down to the nanowire limit," Phys. Rev. B 99, 220506 (2019).

23 G. Yang, P. Stano, J. Klinovaja, and D. Loss, "Majorana bound states in magnetic skyrmions," Phys. Rev. B 93, 224505 (2016). 
${ }^{24}$ U. Güngördü, S. Sandhoefner, and A. A. Kovalev, "Stabilization and control of Majorana bound states with elongated skyrmions," Phys. Rev. B 97, 115136 (2018).

25 M. Garnier, A. Mesaros, and P. Simon, "Topological superconductivity with deformable magnetic skyrmions," Commun. Phys. 2, 126 (2019).

26 N. Mohanta, A. Taraphder, E. Dagotto, and S. Okamoto, "Magnetic switching in Weyl semimetal-superconductor heterostructures," Phys. Rev. B 102, 064506 (2020).

27 E. Mascot, J. Bedow, M. Graham, S. Rachel, and D. K. Morr, "Topological Superconductivity in Skyrmion Lattices," arXiv:2005.00027 (2020).

28 J. Bedow, E. Mascot, T. Posske, G. S. Uhrig, R. Wiesendanger, S. Rachel, and D. K. Morr, "Topological superconductivity induced by a triple-q magnetic structure," Phys. Rev. B 102, 180504 (2020).

29 N. Mohanta, E. Dagotto, and S. Okamoto, "Topological Hall effect and emergent skyrmion crystal at manganiteiridate oxide interfaces," Phys. Rev. B 100, 064429 (2019).

30 N. Mohanta, S. Okamoto, and E. Dagotto, "Planar topological Hall effect from conical spin spirals," Phys. Rev. B 102, 064430 (2020).

31 N. Mohanta, A. D. Christianson, S. Okamoto, and E. Dagotto, "Signatures of a liquid-crystal transition in spin-wave excitations of skyrmions," Commun. Phys. 3, 229 (2020).

32 U. K. Rößler, A. N. Bogdanov, and C. Pfleiderer, "Spontaneous skyrmion ground states in magnetic metals," Nature 442, 797 (2006).

33 S. Mühlbauer, B. Binz, F. Jonietz, C. Pfleiderer, A. Rosch, A. Neubauer, R. Georgii, and P. Böni, "Skyrmion lattice in a chiral magnet," Science 323, 915 (2009).

${ }^{34}$ X. Z. Yu, Y. Onose, N. Kanazawa, J. H. Park, J. H. Han, Y. Matsui, N. Nagaosa, and Y. Tokura, "Real-space observation of a two-dimensional skyrmion crystal," Nature 465, 901 (2010).

35 N. Mohanta, A. P. Kampf, and T. Kopp, "Emergent momentum-space Skyrmion texture on the surface of topological insulators," Sci. Rep. 7, 45664 (2017).

${ }^{36}$ L. Sun, R. X. Cao, B. F. Miao, Z. Feng, B. You, D. Wu, W. Zhang, An Hu, and H. F. Ding, "Creating an artificial two-dimensional Skyrmion crystal by nanopatterning," Phys. Rev. Lett. 110, 167201 (2013).

37 Ö. Gül, H. Zhang, F. K. de Vries, J. van Veen, K. Zuo, V. Mourik, S. Conesa-Boj, M. P. Nowak, D. J. van Woerkom, M. Quintero-Pérez, M. C. Cassidy, A. Geresdi, S. Koelling, D. Car, S. R. Plissard, E. P. A. M. Bakkers, and L. P. Kouwenhoven, "Hard Superconducting Gap in InSb Nanowires," Nano Lett. 17, 2690 (2017).

38 M. T. Deng, S. Vaitiekenas, E. B. Hansen, J. Danon, M. Leijnse, K. Flensberg, J. Nygård, P. Krogstrup, and C. M. Marcus, "Majorana bound state in a coupled quantum-dot hybrid-nanowire system," Science 354, 1557 (2016).

39 B. Nedniyom, R. J. Nicholas, M. T. Emeny, L. Buckle, A. M. Gilbertson, P. D. Buckle, and T. Ashley, "Giant enhanced g-factors in an InSb two-dimensional gas," Phys. Rev. B 80, 125328 (2009).

${ }^{40}$ F. Qu, J. van Veen, F. K. de Vries, A. J. A. Beukman, M. Wimmer, W. Yi, A. A. Kiselev, B.-M. Nguyen, M. Sokolich, M. J. Manfra, F. Nichele, C. M. Marcus, and L. P. Kouwenhoven, "Quantized conductance and large gfactor anisotropy in InSb quantum point contacts," Nano Lett. 16, 7509 (2016).
$41 a$ is the lattice grid spacing used to discretize the kinetic energy term $\frac{p^{2}}{2 m}$ within finite-difference approximation. It is immaterial as long as the lattice lengths remain fixed.

42 D. Sticlet, C. Bena, and P. Simon, "Spin and Majorana polarization in topological superconducting wires," Phys. Rev. Lett. 108, 096802 (2012).

43 S. Głodzik, N. Sedlmayr, and T. Domański, "How to measure the Majorana polarization of a topological planar josephson junction," Phys. Rev. B 102, 085411 (2020).

44 B. Scharf, F. Pientka, H. Ren, A. Yacoby, and E. M. Hankiewicz, "Tuning topological superconductivity in phasecontrolled Josephson junctions with Rashba and Dresselhaus spin-orbit coupling," Phys. Rev. B 99, 214503 (2019).

45 K. Sengupta, I. Žutić, H.-J. Kwon, V. M. Yakovenko, and S. Das Sarma, "Midgap edge states and pairing symmetry of quasi-one-dimensional organic superconductors," Phys. Rev. B 63, 144531 (2001).

${ }^{46}$ L. Kuerten, C. Richter, N. Mohanta, T. Kopp, A. Kampf, J. Mannhart, and H. Boschker, "In-gap states in superconducting $\mathrm{LaAlO}_{3} / \mathrm{SrTiO}_{3}$ interfaces observed by tunneling spectroscopy," Phys. Rev. B 96, 014513 (2017).

47 A. Soumyanarayanan, M. Raju, A. L. Gonzalez Oyarce, Anthony K. C. Tan, M.-Y. Im, A. P. Petrović, Pin Ho, K. H. Khoo, M. Tran, C. K. Gan, F. Ernult, and C. Panagopoulos, "Tunable room-temperature magnetic Skyrmions in $\mathrm{Ir} / \mathrm{Fe} / \mathrm{Co} / \mathrm{Pt}$ multilayers," Nat. Mater. 16, 898 (2017).

48 E. Skoropata, J. Nichols, J. M. Ok, R. V. Chopdekar, E. S. Choi, A. Rastogi, C. Sohn, X. Gao, S. Yoon, T. Farmer, R. D. Desautels, Y. Choi, D. Haskel, J. W. Freeland, S. Okamoto, M. Brahlek, and H. N. Lee, "Interfacial tuning of chiral magnetic interactions for large topological Hall effects in $\mathrm{LaMnO}_{3} / \mathrm{SrIrO}_{3}$ heterostructures," Sci. Adv. 6, eaaz3902 (2020).

49 R. Lo Conte, A. K. Nandy, G. Chen, A. L. Fernandes Cauduro, A. Maity, C. Ophus, Z. Chen, A. T. N'Diaye, K. Liu, A. K. Schmid, and R. Wiesendanger, "Tuning the properties of zero-field room temperature ferromagnetic Skyrmions by interlayer exchange coupling," Nano Lett. 20, 4739 (2020).

50 N. Mohanta and A. Taraphder, "Topological superconductivity and Majorana bound states at the $\mathrm{LaAlO}_{3} / \mathrm{SrTiO}_{3}$ interface," EPL (Europhysics Letters) 108, 60001 (2014).

51 N. Mohanta, A. P. Kampf, and T. Kopp, "Supercurrent as a probe for topological superconductivity in magnetic adatom chains," Phys. Rev. B 97, 214507 (2018).

\section{Acknowledgements}

This work was supported by the U.S. Department of Energy (DOE), Office of Science, Basic Energy Sciences (BES), Materials Sciences and Engineering Division.

\section{Author contributions}

N.M. and E.D. planned the project. N.M. performed the calculations. S.O. provided theoretical inputs. All authors discussed the results, wrote and reviewed the manuscript.

\section{Competing interests}

The authors declare no competing interests. 


\section{Figures}
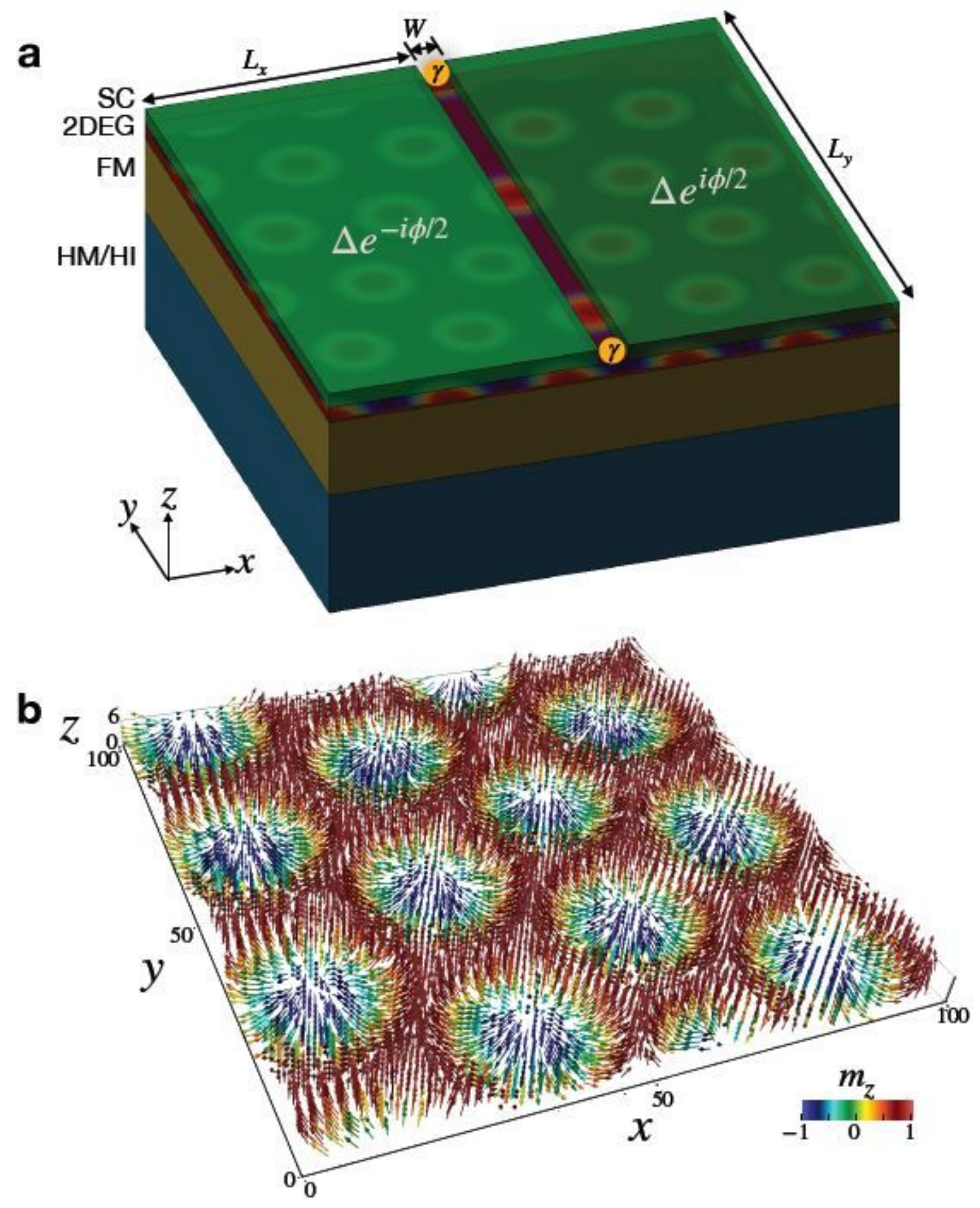

\section{Figure 1}

Device geometry and a skyrmion crystal. a. Planar Josephson junction on top of a skyrmion crystal (SkX). The two-dimensional electron gas (2DEG) exhibits both proximity-induced superconductivity from the top superconductor (SC) layers and spatially-varying magnetism from the bottom SkX. The SkX is 
spontaneously created in the ferromagnet (FM) due to the competition between exchange interactions in the FM and the heavy metal or heavy insulator $(\mathrm{HM} / \mathrm{HI})$, with a field or anisotropy. The zeroenergy Majorana bound states (yellow bubbles) are localized the two ends of the quasi-one-dimensional metallic channel. b. The SkX spin texture, spontaneously generated in a Monte Carlo simulation using a $100 \otimes 100 \otimes 6$ lattice with ferromagnetic exchange interaction strength $J=1$, DMI strength $D=0: 3 \mathrm{~J}$, magnetic field $\mathrm{Hz}=0: 1 \mathrm{~J}$, spin amplitude $S=1$, and easy-plane anisotropy $A=0: 01 \mathrm{~J}$.

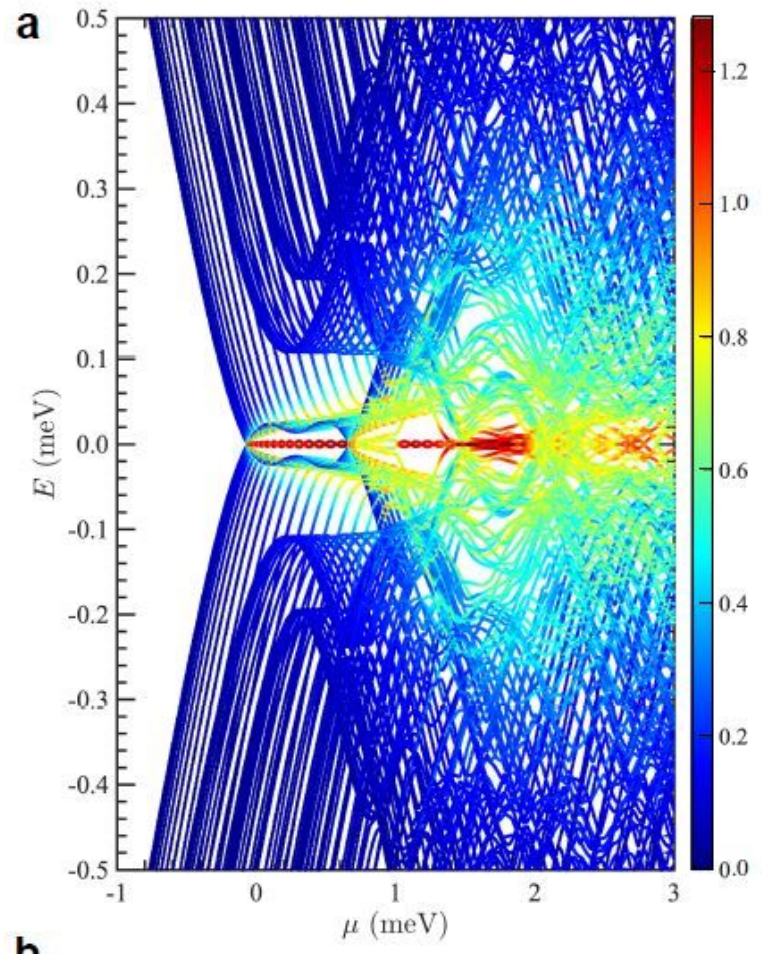

b

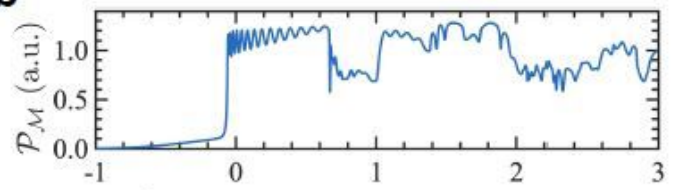

c

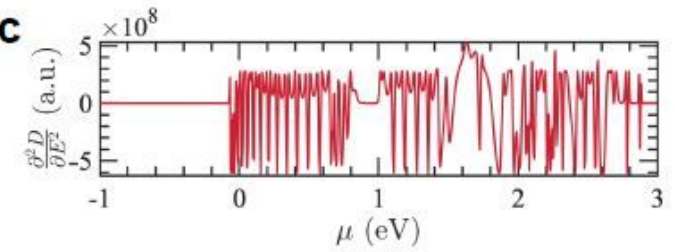

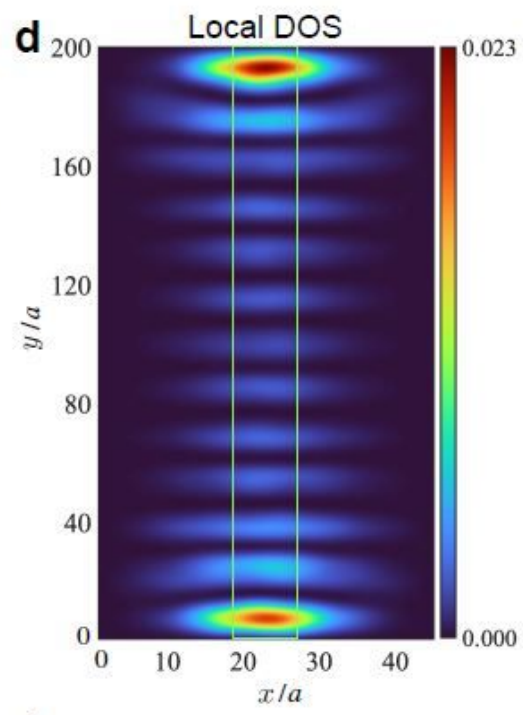
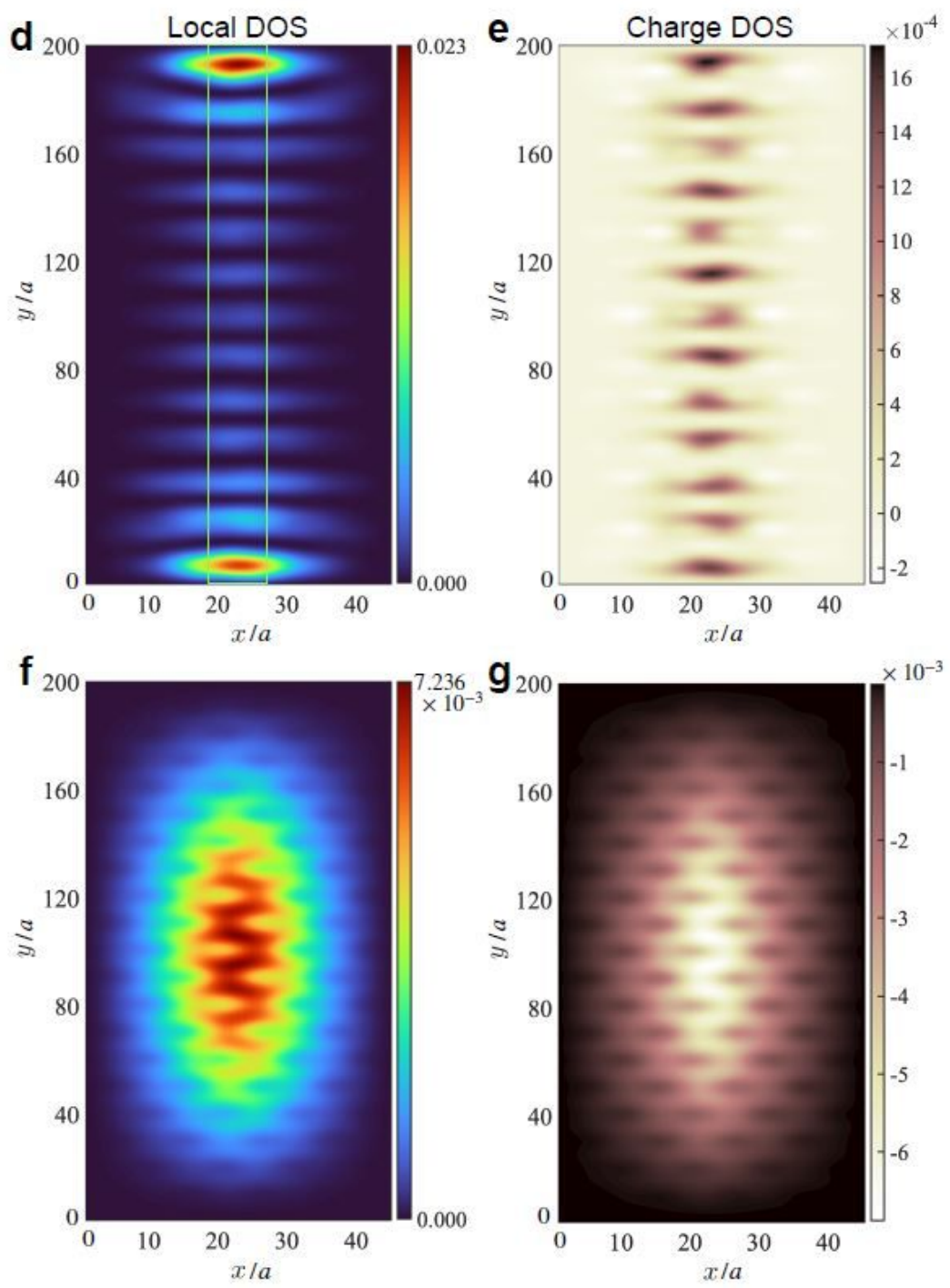

\section{Figure 2}

"Please see the Manuscript PDF file for the complete figure caption". 

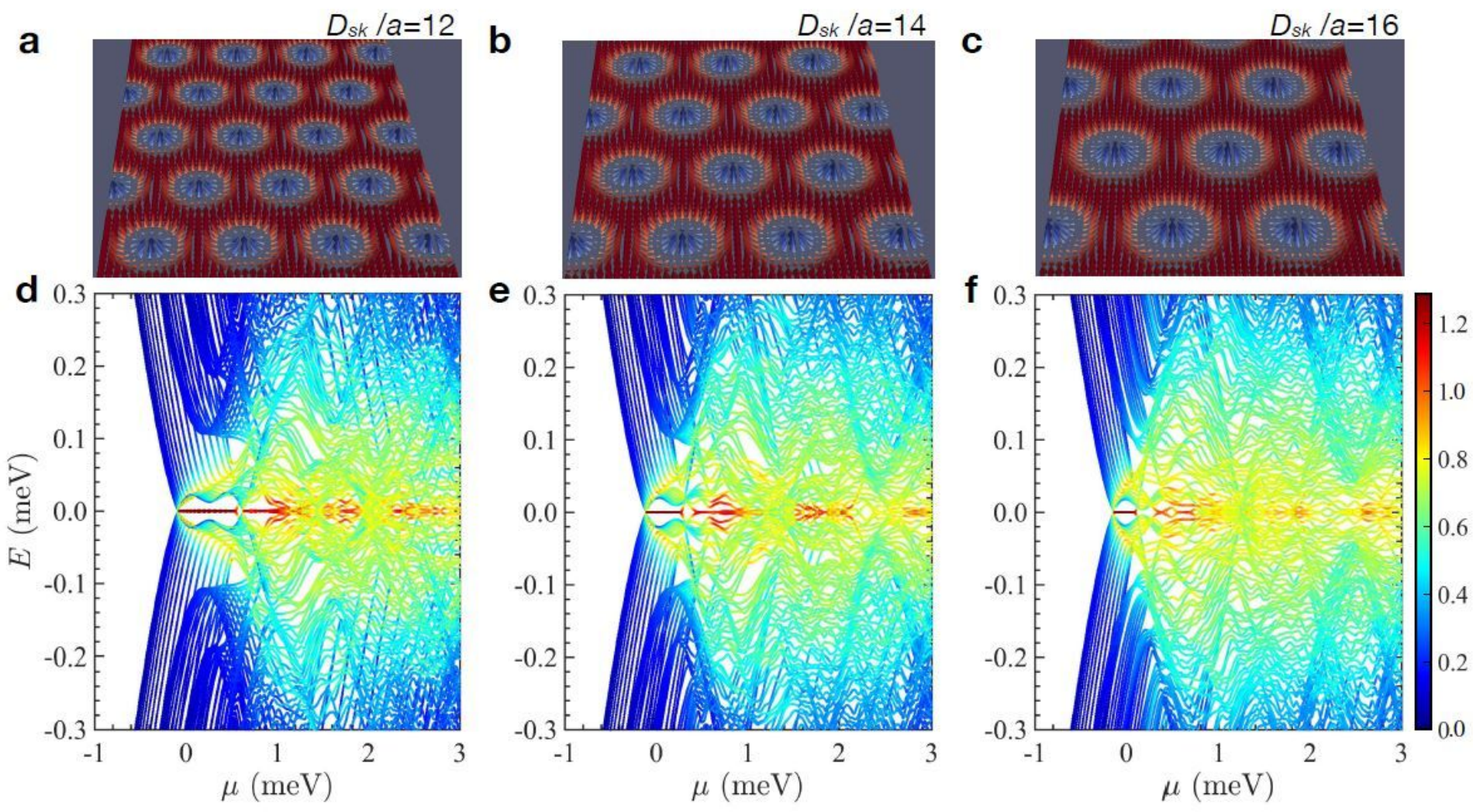

\section{Figure 3}

Skyrmion tuning of the Majorana bound states. a-c The skyrmion crystals of different skyrmion diameters a. Dsk $=12 \mathrm{a}, \mathrm{b}$. Dsk $=14 \mathrm{a}, \mathrm{c}$. Dsk $=16 \mathrm{a}$, obtained in the Monte Carlo calculations using a. a magnetic field $\mathrm{Hz}=0: 8 \mathrm{~J}$, a DMI strength $\mathrm{D}=1: 4 \mathrm{~J}, \mathrm{~b} . \mathrm{Hz}=0: 45 \mathrm{~J}, \mathrm{D}=\mathrm{J}$, and $\mathrm{c} . \mathrm{Hz}=0: 23 \mathrm{~J}, \mathrm{D}=0: 6 \mathrm{~J}$. d-f The corresponding quasiparticle spectra of the planar Josephson junction at the phase difference $\varphi=0$ with varying chemical potential, obtained by solving the Bogoliubov-de Gennes equations with the above skyrmion crystal spin configurations. The colorbar in d-f represents the Majorana polarization PM of the quasiparticle states. 

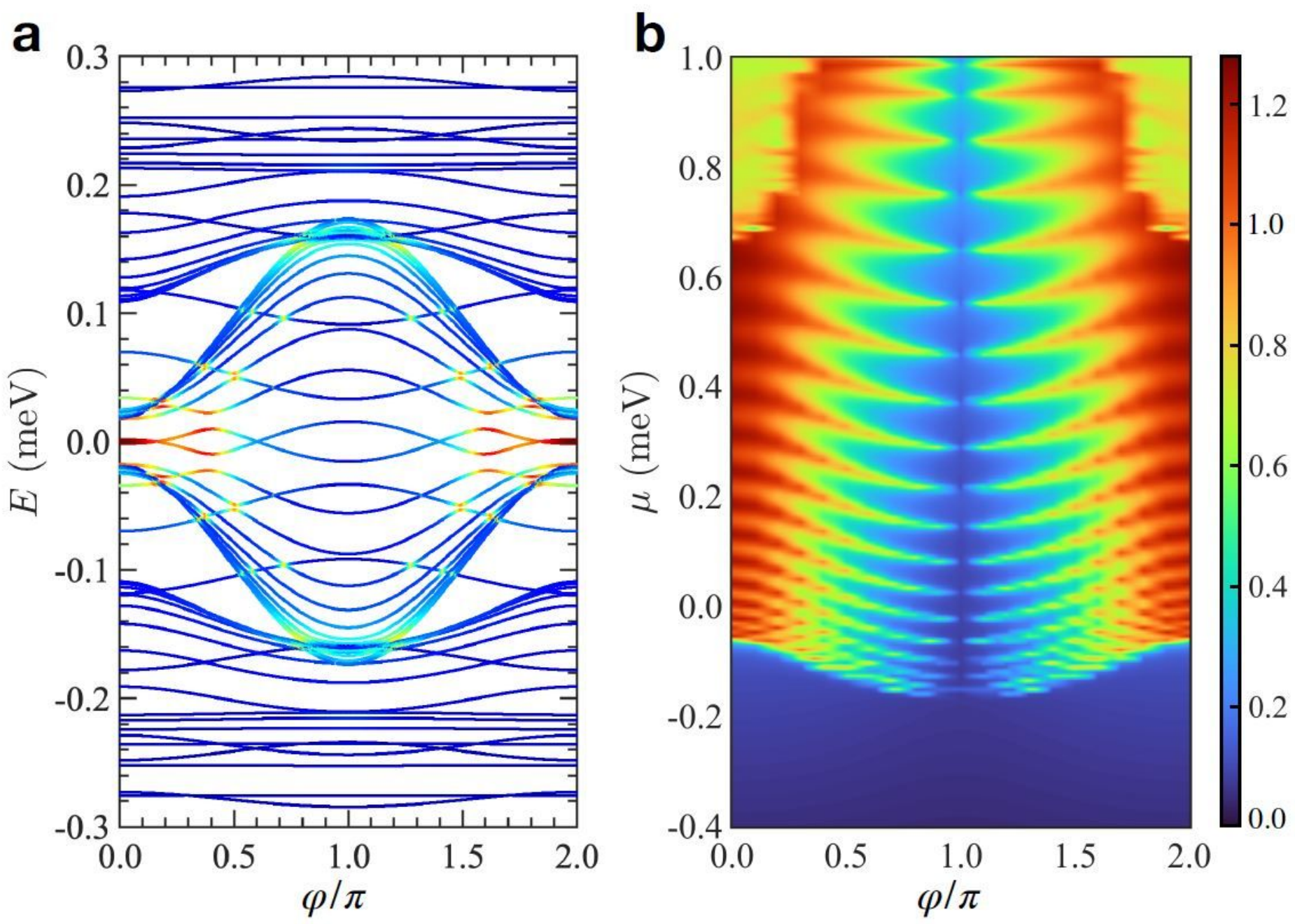

Figure 4

Phase tuning of the Majorana bound states. a. Quasiparticle spectrum of the planar Josephson junction at a chemical potential $\mu=0: 5 \mathrm{meV}$ with changing phase difference $\varphi$ between the two superconducting regions. The skyrmion diameter of the skyrmion crystal was Dsk=10a. b. Colormap of the Majorana polarization of the lowest positive energy state in the plane spanned by the phase difference $\varphi$ and the chemical potential $\mu$. The Majorana bound states appear in the parameter regimes with $P M \geq 1 \varphi=0$ being the most favorable scenario in this set up.

\section{Supplementary Files}

This is a list of supplementary files associated with this preprint. Click to download.

- SupplementaryInformation.pdf 